\title{
Where to live in Lisbon: urban habitat used by the introduced Italian wall lizard (Podarcis siculus)
}

\author{
Ricardo Ribeiro*, Paulo Sá-Sousa \\ ICAAM - Instituto de Ciências Agrárias e Ambientais Mediterrânicas, Universidade de Évora, Pólo da Mitra, \\ Ap. 94, 7002-554 Évora, Portugal \\ *Correspondence: E-mail: ricardoavribeiro@gmail.com
}

Received: 29 March 2018; returned for review: 22 May 2018; accepted 11 September 2018.

Exotic animal invasions constitute a major threat to biodiversity worldwide._Our assessment determined the core range occupied by the Italian wall lizard (Podarcis siculus) in the urban area of Parque das Nações (Lisbon, Portugal), where it was accidentally introduced two decades ago. Despite the apparent current scenario of non-expansion, the alien species interferes with the local distribution pattern of the native $P$. virescens, with both lizard species shown to use the available microhabitats differently. The native $P$. virescens population displays an overall heterogeneous distribution in the urban matrix. On the other hand, the occupancy of the exotic species clusters within the original area of introduction (garden in front of the Lisbon Oceanarium), as well as in adjacent gardens. There, $P$. siculus replaced the native $P$. virescens as the only lizard species present. Appa-rently, there is no known reason there that prevents the alien lizard from colonizing more available geographic area and expanding.

Key words: Invasive species; Podarcis siculus; Podarcis virescens; spatial segregation.

Invasive species currently represent one of the major problems in nature conservation (Schulte, 2012). The damage caused by invasive reptiles mainly comes from disruptions and changes in native food webs and ecosystem functions, with impacts ranging from predation on sensitive species, intoxication of predators, competition and hybridization with native species, vectorization of new parasites, as well as direct and indirect impacts on humans (Kraus, 2009; Fujisaki et al., 2010). Among the European reptile species, the Italian wall lizard (Podarcis siculus, Sauria: Lacertidae) is considered as both an opportunistic species and a successful exotic colonizer, characterized by wide eco- logical tolerance and plasticity, allied with high propagation capacity (Nevo et al., 1972; Capula \& Ceccarelli, 2003; CrnobrNJA-Isailovic et al., 2009; Silva-Rocha et al., 2014). Podarcis siculus is distributed conti -nuously throughout the Italian Peninsula and East Adriatic coast, as well as for big islands - Sardinia (where recent studies suggested that it may be allochtonous instead of native, SENCzuk et al., 2017) and Sicily - and a multitude of adjacent islets (Corti \& Lo CAscio, 2002). Unlike the reported pet trade introductions in the USA (Kolbe et al., 2013), it might have been introduced by ship trade into Corsica, Balearics and several port areas around the Mediterranean Basin e.g. SW France, 
Greece, Turkey, Tunisia or Lybia (CARretero \& Silva-Rocha, 2015).

Towards Lisbon, some P. siculus individuals might have been transported along with rough materials and ornamental plants (structures in which they would most likely be sheltered in or basking on), from their place of origin, during the Expo98 event (González de la Vega et al., 2001; Silva-Rocha et al., 2012). This appears to be a recurrent issue, as similar cases were reported by Rivera et al. (2011) and VALDEÓN et al. (2010), after the observation of several $P$. siculus individuals in olive trees, imported from Calabria (Italy) to Catalonia (Spain).

In urban environments, which are typically characterized by spatially and temporally heterogeneous thermal mosaics, ecological interactions with potential competitors and predators may also influence patterns of occurrence and abundance of individuals of a given species (Kolbe et al., 2016). In those environments, it has been observed that sympatric lacertids (namely Podarcis muralis and P. siculus) are spatially organized according to each species specific ecological needs, and therefore not limited to interspecific interactions (CAPula et al., 1993). Lizard species often tend to be associated with specific microhabitat patches, with minimized interspecific competition through their spatial niche distribution (SMith \& BaLlinger, 2001; Rugiero \& Luiselli, 2007; VitT \& CALdwell, 2014). However, there may be cases where the microhabitat occupied by a certain species is restricted by the presence of another (ARnOLD, 1987). That could be the case with autochthonous Podarcis virescens (GENIEZ et al., 2014; DiAs et al., 2016), which might be affected by this $P$. siculus introduction, although negative impacts are still unknown (such as expansion, hybridization or extinction of native species). Both species under study coexist in sympatry with other lacertids in their native distributions, evidencing ecological segregation (CAPUla, 1993; CAPUla et al., 1993; CARretero, 2008; VAldeón $e t$ al., 2010; Grano et al., 2011; Carretero \& Silva-Rocha, 2015; Carretero \& Salvador, 2016).

However, the introduction of the Italian wall lizard in other areas has been shown to be detrimental to autochthonous lacertids, especially in micro-insular environments, with evidence of competitive spatial coexistence or exclusion (Nevo et al., 1972; CAPula, 2002; Capula \& Ceccarelli, 2003; PodNAR et al., 2005; VALdeón et al., 2010; Mateo et al., 2011; Carretero \& Silva-Rocha, 2015).

Based on previous personal reports from Paulo Sá-Sousa and bibliography from Loureiro et al. (2008), this study's main objectives consisted in determining the current range area occupied by the introduced $P$. siculus and assessing the degree of spatial segregation exerted by the exotic population upon the autochthonous species. This required the knowledge of the current spatial distribution of both species in Lisbon, as well as each species microhabitat preferences, according to the available space.

\section{Materials AND METhOdS}

The study area covered the location where $P$. siculus was first introduced and consequently reproduced (GonZÁLEz DE LA Vega et al., 2001), within the so-called Parque das Nações, a leisure and residential area of Lisbon $\left(38^{\circ} 45^{\prime} 43.7^{\prime \prime} \mathrm{N} 9^{\circ} 05^{\prime} 39.0^{\prime \prime} \mathrm{W}\right.$, 


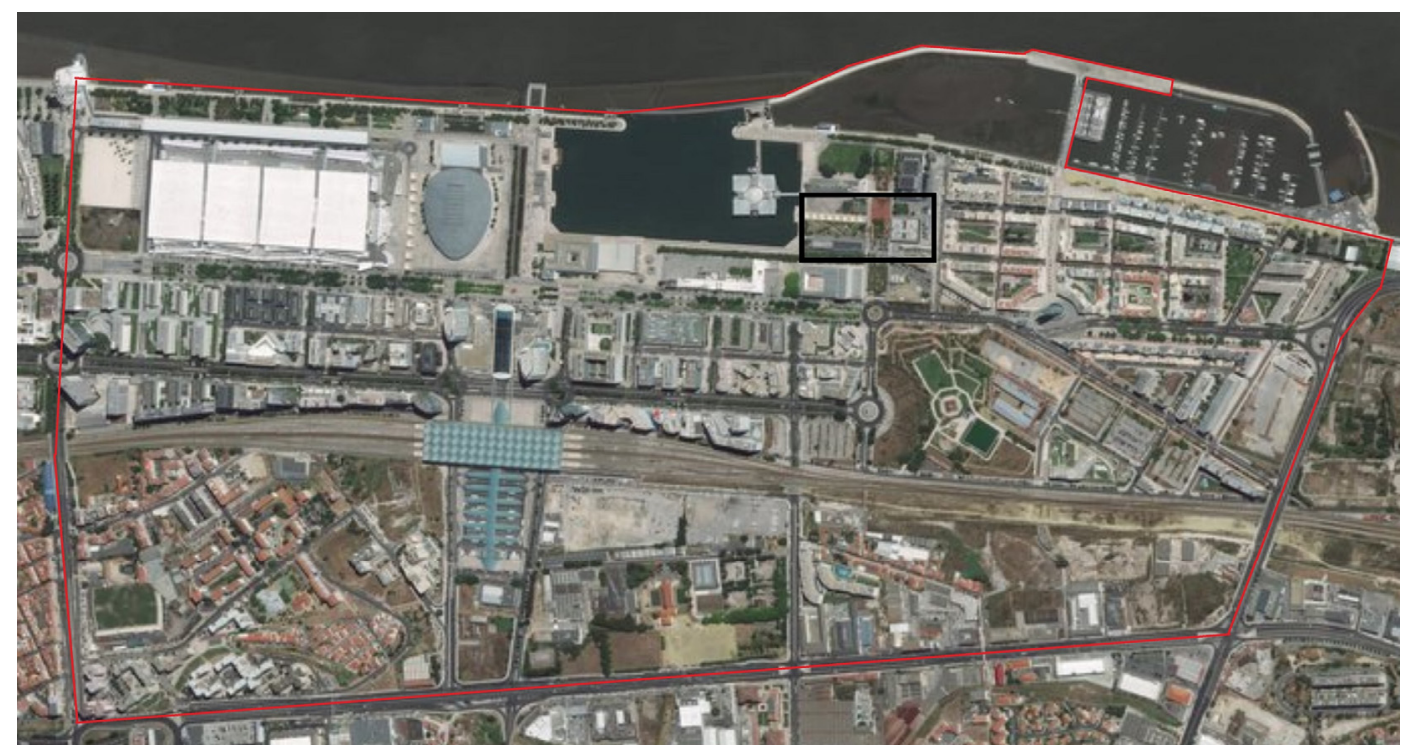

Figure 1: Total prospected area limited in red; Podarcis siculus introduction spot highlighted by a black square.

Fig. 1). For lizard sampling one large polygon was delimited to address the possible expansion and establishment of new populations towards adjacent spots. The sampling area was subsequently divided in 16 plots or sectors with approximate dimensions, to facilitate the visual encounter of lizards. Although these plots consisted of urban areas, there are contrasting differences between them, ranging from those that show predominance of artificial elements, to those that hold some garden spots with lawn or a more rustic appearance, usually consisting of an eclectic selection of plant species from various regions of the world (Pedrosa, 2013).

Due to statistical and data independence reasons, the sectors were sampled following a random order. Each sector was surveyed by walking at a slow, steady pace, during one sunny day (when there were favourable weather conditions for lizard thermoregulation). However, some sectors took longer to cover in cases of high occurrence of observations. All lizard observations (each corresponding to one individual) consisted of visual encounters (LAmbert, 1984; Eекноut, 2010), where the following data was recorded: GPS point of location, species $(P$. siculus or $P$. virescens), behaviour activity, perch type, height and slope, distance to vegetation cover, refuge type and distance to refuge, along with the substrate texture, number of trees, shrubs, non-woody plants and dead branches/trunks, found within a four meters radius surrounding each observation point (the dissertation from which this article was adapted covered a wider range of variables, including sex and size class, which were considered negligible for the study's purpose, primarily focused on 


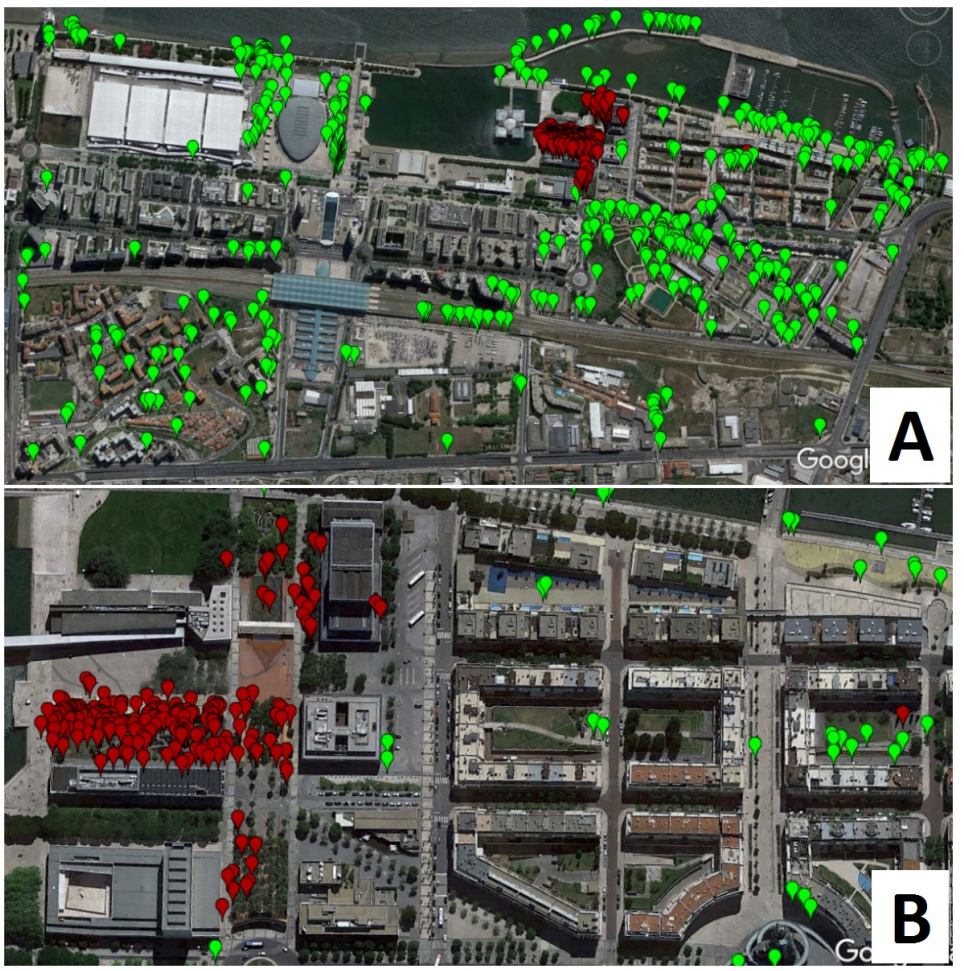

Figure 2: Spatial distribution of both species (A - total area; B - focus in the presence of Podarcis siculus). Red symbols - introduced Podarcis siculus; Green symbols - autochthonous Podarcis virescens.

habitat use and microspatial distribution, and therefore discarded from this paper). The first round of sampling covered all (16) sectors, providing a general acquaintance of both the study area and its lizard occupancy. Based on this previous data assessment, the second and third rounds covered only 8 of the 16 sectors, excluding those where $P$. siculus was clearly absent in the first round. Thus, the first round took place between April 25 th 2016 and July 3 $3^{\text {rd }}, 2016$; the second began on September $8^{\text {th }}, 2016$ and ended on September 21 st, 2016; the third lasted from September $24^{\text {th }}$, 2016 to October $11^{\text {th }}$, 2016.

Further statistical analyses were performed using the SigmaPlot ${ }^{\circledR}$ 13.0, Statistica 13® and Microsoft Excel 2016 softwares, tested for a confidence level of 95\% ( $\alpha=$
0.05) (Strode \& Brokaw, 2015). The chisquared test was used for comparison between species, regarding the data collected on the constituent elements of the microhabitats used by both species, where the formulated null hypothesis was that "there are no differences between the use given by the invasive and autochthonous species to the elements present in the microhabitat". For this test's application we used raw data, that is, simple counts (observations), instead of percentages or proportions (STRODE \& BROKAw, 2015), presented in table, which included the observed and expected values for several categories, within each considered parameter (Dутнам, 2011). Since multiple tests were performed simultaneously, further statistical corrections were made using the Holm-Bonferroni method, 
Table 1: Sampled sector maximums for both species in each sector. The three sectors with occurrence of both species highlighted in grey.

\begin{tabular}{ccc} 
Sector & Max. P. virescens & Max. $P$. siculus \\
\hline 1 & 26 & 1 \\
2 & 9 & 26 \\
3 & 22 & 96 \\
4 & 41 & 0 \\
5 & 13 & 0 \\
6 & 7 & 0 \\
7 & 4 & 0 \\
8 & 8 & 0 \\
9 & 27 & 0 \\
10 & 24 & 0 \\
11 & 2 & 0 \\
12 & 10 & 0 \\
$13 / 14$ & 33 & 0 \\
15 & 9 & 0 \\
16 & 16 & 0 \\
Sub-total & $\mathbf{2 5 1}$ & $\mathbf{1 2 3}$ \\
\hline
\end{tabular}

narrowing the possibility of generating a statistically significant result (type I error) (Holm, 1979). To enlighten these intricate multiple results, a Multiple Correspondence Analysis test was performed comprehending all parameters, to visualize the pattern of relationships between the microhabitat features and their usage by both species (Abdi \& VAlentin, 2007).

\section{Results}

\section{Lizard occupancy}

A total of 699 observations were registered, $476(68 \%)$ belonging to the native $P$. virescens and $223(32 \%)$ to the introduced $P$. siculus, present in only three sectors (Table 1 ).
The native $P$. virescens population shows an overall heterogeneous distribution in the urban matrix. On the other hand, the occupancy of the invasive species only clusters within the original area of introduction (garden in front of the Lisbon Oceanarium), as well as in adjacent gardens (Fig. 2a). A single individual of $P$. siculus was also o-bserved in a previously unknown location (a garden spot placed some hundreds of meters away from the main area), where $P$. virescens often occurs (Fig. 2b).

\section{Degree of spatial segregation between} Podarcis siculus and Podarcis virescens

The Chi-squared tests of independence support the main differences found in the spatial variables tested between $P$. siculus and $P$. virescens, as explained in the following sections concerning habitat, through the analysis of the graphics and tables $(P<$ 0.001 for nearly all values, Table 2$)$, regarding the behaviour shown by the lizards during observation, the elements present in the microhabitat and its use by both species. The only exception was the parameter "Distance to Refuge", which was similar for both species $(P=0.223$, Table 2$)$. The HolmBonferroni correction method confirmed the obtained results, whereas the previously mentioned parameter remained as the only to not reject the null hypothesis.

The Multiple Correspondence Analysis test plots for both species (Fig. 6) showed that, despite overlapping for the most part, $P$. siculus only occupies part of the spatial niche of $P$. virescens at the multivariate level, which could provide some answers regarding the possible event of future expansion and its impacts on $P$. virescens. 
Table 2: Results of the chi-Squared statistical tests, used for comparison between species. Significant $P$-values highlighted in bold. Adjusted Holm-Bonferroni $P$-values on the right.

\begin{tabular}{lllll}
\hline Tested Parameters & $\chi^{2}$ & df & $P$-value & $\begin{array}{l}\text { Holm-Bonferroni } \\
\text { values }\end{array}$ \\
\hline Behaviour & 19.401 & 3 & $\boldsymbol{P}<\mathbf{0 . 0 0 1}$ & $P=0.01$ \\
Perch type & 81.089 & 3 & $\boldsymbol{P}<\mathbf{0 . 0 0 1}$ & $P=0.005$ \\
Perch height & 51.731 & 3 & $\boldsymbol{P}<\mathbf{0 . 0 0 1}$ & $P=0.03$ \\
Perch slope & 22.236 & 2 & $\boldsymbol{P}<\mathbf{0 . 0 0 1}$ & $P=0.01$ \\
Refuge type & 98.363 & 6 & $\boldsymbol{P}<\mathbf{0 . 0 0 1}$ & $P=0.01$ \\
Distance to refuge & 4.379 & 3 & $P=0.223$ & $P=0.01$ \\
Number of Trees & 34.2 & 1 & $\boldsymbol{P}<\mathbf{0 . 0 0 1}$ & $P=0.02$ \\
Number of Shrubs & 43.719 & 2 & $\boldsymbol{P}<\mathbf{0 . 0 0 1}$ & $P=0.01$ \\
Number of non-woody plants & 12.945 & 2 & $\boldsymbol{P}=\mathbf{0 . 0 0 2}$ & $P=0.05$ \\
Number of dead branches/trunks & 33.585 & 3 & $\boldsymbol{P}<\mathbf{0 . 0 0 1}$ & $P=0.004$ \\
Substrate texture & 62.558 & 2 & $\boldsymbol{P}<\mathbf{0 . 0 0 1}$ & $P=0.01$ \\
Distance to vegetation cover & 21.584 & 2 & $\boldsymbol{P}<\mathbf{0 . 0 0 1}$ & $P=0.01$ \\
\hline
\end{tabular}

Among the variables which contributed the most for the relative inertia values, were the Refuge and Perch Type Rock, as well as Vertical Perch Slope, and Artificial Perch Type and Substrate Texture.

\section{Habitat use}

Both species seem to occur in larger numbers $(84 \%$ of the $P$. siculus total and $97 \%$ of $P$. virescens) in areas with few or no trees (0 to 2 ) (Fig. 3a), with the autochthonous species being less dependent on the existence of arboreal and non-woody plants cover on site (Fig. 3c - the congeners showed a marked preference for areas without non-woody plants, $73 \%$ of $P$. siculus and $80 \%$ P. virescens). Similarly, $58 \%$ of $P$. siculus and $56 \%$ of $P$. virescens individuals appeared in spots without shrubs (Fig. 3b) but were also present in areas with 1 to 5 (23\% and $39 \%$ ) shrubs. Both species used areas with a moderate presence of dead wooden matter (up to 30 branches/trunks/ etc.), counting for $71 \%$ of $P$. siculus and $56 \%$ of $P$. virescens observations (Fig. 3d). Substrate texture (Fig. 3e) enhances $P$. siculus as having occupation for earthy open spaces (79\%), while $P$. virescens appears to be a more generalist species, though often found in earthy substrate $(50 \%)$, dividing the remaining half of observations almost equally between artificial and mixed grounds.

\section{Micro-habitat features}

The most used perch for both lacertids (Fig. 4a) appears to be the ground (more evident for $P$. siculus, $81 \%$, than for P. virescens, 53\%), whereas $P$. virescens also choos- 
A

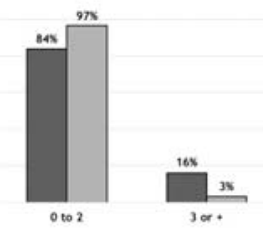

C

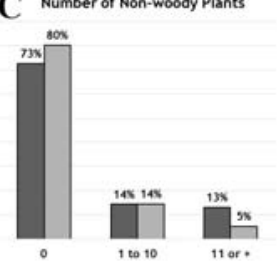

E Substrate Texture

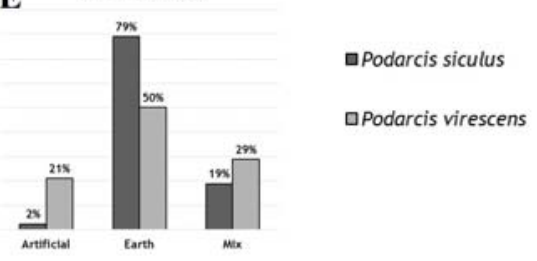

Figure 3: Results concerning micro-habitat elements: number of Trees (A), Shrubs (B), Non-woody Plants (C), dead Branches/Trunks (D) and Substrate Texture (E).

es perches located in artificial stru-ctures (34\%). The rocky perches were the least chosen, by both species. Concerning to the perch height, soil level was the most observed (Fig. 4b), with P. siculus showing ground dwelling attributes (82\%), similar to slightly more than half of the $P$. virescens individuals. Of the latter, $21 \%$ are still distributed at a reduced height (from 0 to $0.25 \mathrm{~m}$ ), with only $9 \%$ of the individuals found at heights above $0.75 \mathrm{~m}$. The slope assumed by each lizard at perch site (Fig. 4c) was mostly horizontal for both species ( $84 \%$ of $P$. siculus and $70 \%$ of P. virescens). The "Vertical" posture was the second most observed in $P$. virescens and the least observed in P. siculus. High dependency
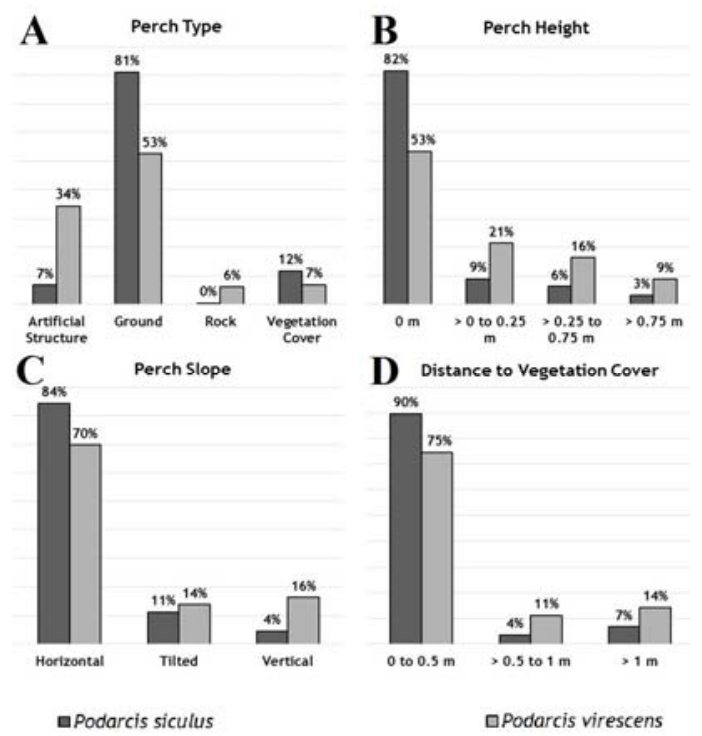

Figure 4: Results concerning each species use of the available micro-habitat: Perch Type (A), Height (B), Slope (C) and Distance to Vegetation Cover (D).

(90\% for $P$. siculus and $75 \%$ for $P$. virescens) of vegetation cover (herbaceous, shrubs, trees - Fig. 4d) near to the perch (up to $0.5 \mathrm{~m}$ ) was detected.

The behaviour activity of lizards (Fig. 5a) shows that both species were often seen basking (57\% for $P$. siculus and $64 \%$ for $P$. virescens), but also active (18 to $20 \%$ ), suggesting a similar daily tasks pattern. When disturbed, the lizards often seek the available vegetation cover as refuge (Fig. 5b), which was evident (50\%) for $P$. siculus, while the native lizard chose between ve-getation cover and artificial structures ( $26 \%$ and $32 \%$, respectively), the latter one which the exotic species apparently tends to avoid. Approximately $22 \%$ 
A

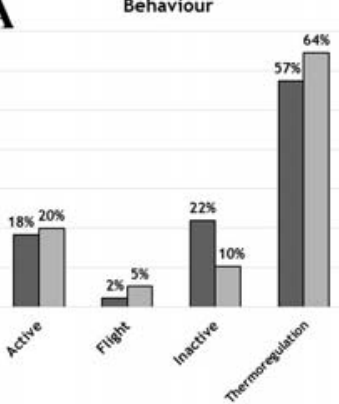

-Podarcis siculus

口Podarcis virescens
Figure 5: Results concerning each species use of the available micro-habitat: Behaviour (A), Refuge Type (B) and Distance to Refuge (C).

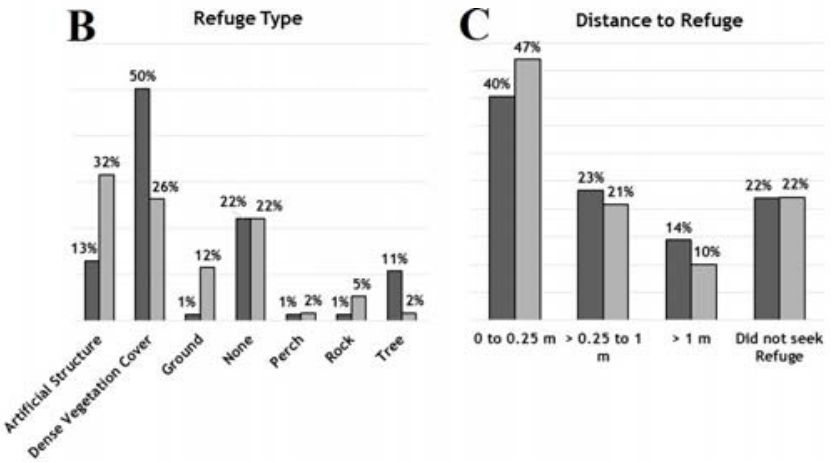

of the observations for both species did not seek refuge, remaining in the perch without mo-ving, or escaping without seeking shelter in the nearby elements. About $40 \%$ of $P$. siculus and $47 \%$ of $P$. virescens prefer to carry out their daily tasks near (up to $0.25 \mathrm{~m}$ ) to a possible refuge (Fig. 5c), with the number of observations decreasing as the distance to refuge increases.

\section{Discussion}

Although there were found some significant differences in the use of several categories of habitat tested between both lizard species, that is not the major evidence. Indeed, all observations in Parque das Nações (Lisbon) point to a scenario of absence of expansion by the alien species $P$. siculus, as previously suggested by LoureIro et al. (2008) and Sá-Sousa's personal ob- servations. There, this species individuals show the behaviour and ecological patterns typical of their own species (Corti \& Lo Cascio, 2002). However, the obtained data suggests that local spatial segregation was most probably due to competitive exclusion, as $P$. siculus replaced $P$. virescens as the only lizard species present at the original introduction site. In fact, $P$. siculus has a larger body size than $P$. virescens, which is probably an advantage in competitive encounters since, according to Downes \& Bauwens (2002), larger species tend to be dominant over smaller ones. This appears to have favoured the exotic wall lizard to the detriment of the native species, since in Lisbon the species are shown to be sympatric but never syntopic.

Sympatric species, when in direct competition, often spatially separate them- 


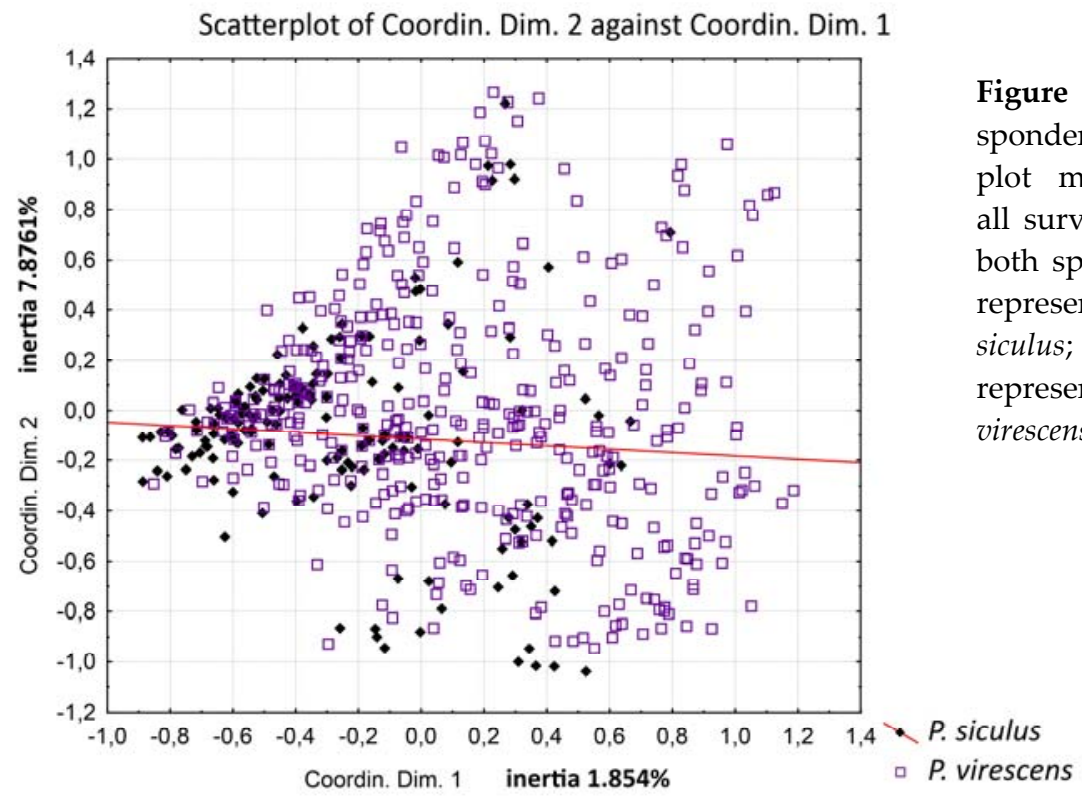

selves due to differences in the structural niche (e.g. diameter and height of the chosen perch) and, in some cases, in the microclimatic environment (eg. open vs. shaded areas) or by displacement of individuals through both competition and anti -predatorial/survival behaviour (Downes \& BAuwens, 2002; ŽAGAR et al., 2015). Syntopic lizard populations were shown to alter their predation risk by using specific habitats based on the availability of refu-ges that provide protection against shared predators, which act as competition mediators, indirectly inducing asymmetrical competition and shifts in segregation patterns (SMITH \& BALlinger, 2001; ŽAGAR et al., 2015). The results in Lisbon suggest that both species use the available microhabitats differently.

The exotic species $P$. siculus preferentially uses microhabitats having a mostly earthy substrate texture, avoiding fully artificial substrates. It shows, to some extent, dependence on the existence of some type of vegetation cover (herbaceous, shrubs, trees) relatively close to the perch, being mainly found at soil level. Despite its observable proximity to vegetation cover, the microhabitats used by $P$. siculus tend to be poor in tree and shrub cover, with most of the individuals observed also showing preference for areas with little to none dead wooden matter on the ground. It was also observed, in this species, tendency to avoid artificial structures as possible shelter, preferring the available vegetation cover for this purpose - and thus perhaps limiting its distribution in this predominantly urban area.

It is tricky to explain the present lack of expansion of P. siculus in Lisbon, especially since this species often spreads into urban areas in native Italy, where it is sympatric with $P$. muralis: $P$. siculus prefers the 
driest places, while $P$. muralis the moisten ones (CorTi \& Lo CAscio, 2002). Perhaps it is still a question of a short time lapse (two decades) after the presumed date of introduction (not yet the sufficient time and effort required to efficiently use the newly occupied habitat - STAмPs, 1995), the possibility of the exotic species larger body size being a disadvantage at this particular introduction site (making this lizard more prone to predation or less capable of dealing with the introduction site's conditions), and/or lack of a reproductive effective high enough to promote recruitment of young lizards towards new adjacent spots (more individuals $=$ more potential dispersers - Vignoli et al., 2012).

This diminished reproductive effective might also be due to high predation pressure, as several individuals of Tarentola mauritanica were observed in the $P$. siculus place of occurrence and nearby sites, a species which may consume individuals of the latter and other species of lacertids, both immature and adult (Pellitteri-Rosa et al., 2015). This population growth control may be carried out by $P$. siculus adult individuals as well, in cases of cannibalism (such as those previously described by Ouboter, 1981, Burke \& Mercurio, 2002, Cattaneo, 2005, Capula \& Aloise, 2011 and GRANo et al., 2011), or by cats, dogs or seagulls present in the area (BURKE \& NER, 2005). Other lacertid found inside the study area limits was Psammodromus algirus, which could spark interactions with yet unknown consequences in case of future contact with the exotic population.

Although the introduction area in Parque das Nações is surrounded by artificial structures that apparently provide little or no shelter to these $P$. siculus individuals, they might attempt to expand. If landscape work is carried out in the adjacent areas and gardens, it may possibly promote the dispersal of $P$. siculus, by altering their previously unfavourable conditions and resources, since dispersal behaviour seems to be in some aspects influenced by external factors, such as intraspecific competition and habitat quality (Vignoli et al., 2012). This brings us to the single individual found off the core area of introduction, which may occur most likely due to the translocation of plants or trees between gardens of Parque das Nações, an action witnessed during the sampling rounds. Most of these movements are usually carried out in the winter, due to the reduced plant activity, which facilitates the success of the transplant, but also favours exotic reptile species introductions, often hibernating inside available cavities in trees or plants, so that these movements do not cause the individual to escape from the refuge (VAldeón et al., 2010). Despite the contribution of such activities and regular maintenance work to introductions, they can also rake the soil and diminish the available vegetation cover, destroy lizard eggs buried in the ground or sand, reduce the availability of perches for thermoregulation and disturb the existing microclimate at the place of introduction yet again providing a plausible argument for the absence of expansion. This clustering could be due to high predation pressure, short time span since introduction, competition with autochthonous species, lack of available favourable resources and/ or microhabitat degradation - or synergy of all the previous factors. If expansion is 
to occur, data suggests that it possibly would not exclude the autochthonous species entirely, since $P$. siculus seems to not occupy all the available habitats in the Parque das Nações area, with a reduced usage of the more artificial and/or rocky ones.

Nevertheless, the present study demonstrates how there is no homogeneity of introduction/invasion cases, as naturalized populations can vary enormously in their ecological dominance, from those that remain in small numbers in a single locality, to those that spread like wildfire on a large scale and become numerically dominant (Kraus, 2009). Similarly, for the other known introduced populations of $P$. siculus in the Iberian Peninsula, there is no evidence of high expansion in Almeria, while it seems to be decreasing in Cantabria or even being extirpated from La Rioja and Catalonia (CARretero \& Silva-Rocha, 2015). Since the eradication of alien reptile populations is virtually impossible once established (KraUS, 2009), we suggest the monitoring and containment of the exotic population, as well as planning maintenance works considering the presence of the species, as prevention measures to avoid future territorial expansion and consequent impacts on native fauna.

\section{Acknowledgement}

This article was adapted from Ricardo Ribeiro's master's dissertation in Conservation Biology, titled "First assessment on the introduction of the Italian wall lizard (Podarcis siculus) in Parque das Nações (Lisbon)", supervised by Paulo Sá-Sousa, available in http:// hdl.handle.net/10174/21333. Special thanks to Leonor Melo for the additional review of the article. Miguel A. Carretero and one anonymous reviewer provided constructive comments and suggestions on the manuscript.

\section{ReFERENCES}

Abdi, H., \& VAlentin, D. (2007). Multiple correspondence analysis. Encyclopedia of measurement and statistics, 651-657.

Arnold, E. N. (1987). Resource partition among lacertid lizards in southern Europe. Journal of Zoology 1(4): 739-782.

Burke, R. L., \& Mercurio, R. J. (2002). Food habits of a New York population of Italian wall lizards, Podarcis sicula (Reptilia, Lacertidae). The American Midland Naturalist 147(2): 368-375.

Burke, R. L. \& Ner, S. E. (2005). Seasonal and diel activity patterns of Italian wall lizards, Podarcis sicula campestris, in New York. Northeastern Naturalist 12(3): 349-360.

Capula, M. (1993). Natural hybridization in Podarcis sicula and P. wagleriana (Reptilia: Lacertidae). Biochemical Systematics and Ecology 21(3): 373-380.

Capula, M. (2002). Genetic evidence of natural hybridization between Podarcis sicula and Podarcis tiliguerta (Reptilia: Lacertidae). Amphibia-Reptilia 23(3): 313-321.

Capula, M. \& Ceccarelli, A. (2003). Distribution of genetic variation and taxonomy of insular and mainland populations of the Italian wall lizard, Podarcis sicula. Amphibia -Reptilia 24: 483-495.

Capula, M. \& Aloise, G. (2011). Extreme feeding behaviours in the Italian wall lizard, Podarcis siculus. Acta Herpetologica 6(1): 1114.

Capula, M., Luiselli, L. \& Rugiero, L. (1993). Comparative ecology in sympatric Podarcis muralis and $P$. sicula (Reptilia: Lacertidae) from the historical centre of Rome: What about competition and niche segregation in an urban habitat?. Bolletino di Zoologia 60(3): 
287-291.

Carretero, M. A. (2008). An integrated assessment of a group with complex systematics: the Iberomaghrebian lizard genus Podarcis (Squamata, Lacertidae). Integrative Zoology 4: 247-266.

Carretero, M. A. \& Silva-Rocha, I. (2015). La lagartija italiana (Podarcis sicula) en la Península Ibérica e islas Baleares. Ecology 22: 4829-4841.

Carretero, M. A. \& Salvador, A. (2016). Lagartija verdosa - Podarcis virescens, In A. Salvador \& A. Marco (eds.) Enciclopedia Virtual de los Vertebrados Españoles. Museo Nacional de Ciencias Naturales, Madrid. http://www.vertebradosibericos.org/.

Cattaneo, A. (2005). L'erpetofauna della Tenuta Presidenziale di Castelporziano (Roma). Atti del Museo di Storia Naturale della Maremma 21: 49-77.

Corti, C. \& Lo Cascio, P. (2002). The lizards of Italy and adjacent areas. Frankfurt Contributions to Herpetology, 14 Frankfurt am Main.

Crnobrnja-Isailovic, J., Vogrin, M., Corti, C., Pérez Mellado, V., Sá-Sousa, P., Cheylan, M., Pleguezuelos, J., Sindaco, R., Romano, A. \& Avcr, A. (2009). Podarcis siculus. (errata version published in 2016) The IUCN Red List of Threatened Species 2009: e.T61553A86151752

Dias, G., Luis, C., Pinho, C. \& Kaliontzopoulou, A. (2016). A case of Podarcis carbonelli intake by Podarcis virescens. Herpetology Notes 9: 105-108.

Downes, S. \& BAuwens, D. (2002). An experimental demonstration of direct behavioural interference in two Mediterranean lacertid lizard species. Animal Behaviour 63(6): 1037 $-1046$.

Dyтнам, C. (2011). Choosing and using statistics - a biologist's guide. 3rd ed. WileyBlackwell

Eекноuт, X. (2010). Sampling amphibians and reptiles. Manual on field recording tech- niques and protocols for all taxa biodiversity inventories. Belgium: Belgian National Focal Point to The Global Taxonomy Initiative 8: 530-557.

Fujisaki, I., Hart, K. M., Mazzotti, F. J., Rice, K. G., Snow, S. \& Rochford, M. (2010). Risk assessment of potential invasiveness of exotic reptiles imported to south Florida. Biological Invasions 12(8): 2585-2596.

Geniez, P., Sá-Sousa, P., Guillaume, C. P., Cluchier, A. \& Crochet, P. A. (2014). Systematics of the Podarcis hispanicus complex (Sauria, Lacertidae) III: valid nomina of the western and central Iberian forms. Zootaxa 3794(1): 001-051. DOI: http:// dx.doi.org/10.11646/zootaxa.3794.1.1

González de la Vega, J. P., González-García, J. P., García-Pulido, T. \& González García, G. (2001). Podarcis sicula (Lagartija italiana), primera cita para Portugal. Boletín de la Asociación Herpetológica Española 12(1): 9.

Grano, M., Cattaneo, C. \& Cattaneo, A. (2011). A case of cannibalism in Podarcis siculus campestris De Betta, 1857 (Reptilia, Lacertidae). Biodiversity Journal 2(3): 151152.

Holm, S. (1979). A simple sequentially rejective multiple test procedure. Scandinavian journal of statistics 6(2): 65-70.

Kolbe, J. J., Lavin, B. R., Burke, R. L., Rugiero, L., Capula, M. \& Luiselli, L. (2013). The desire for variety: Italian wall lizard (Podarcis siculus) populations introduced to the United States via the pet trade are derived from multiple native-range sources. Biological Invasions 15(4): 775-783.

Kolbe, J. J., Van Middlesworth, P., Battles, A. C., Stroud, J. T., Buffum, B., Forman, R. T. \& Losos, J. B. (2016). Determinants of spread in an urban landscape by an introduced lizard. Landscape Ecology 31(8): 1795-1813.

Kraus, F. (2009). Alien reptiles and amphibian. A scientific compendium and analysis. Invading nature: Springer series. Invasion Ecology 4. 
Lambert, M. R. K. (1984). Amphibians and reptiles, In J.L. Cloudsley-Thompson (ed.) Sahara Desert. Pergamon Press, Oxford, United Kingdom: 205-228.

Loureiro, A., Ferrand de Almeida, N., Carretero, M. A. \& Paulo, O. S. (2008). Atlas dos anfíbios e répteis de Portugal. Instituto da Conservação da Natureza e da Biodiversidade, Lisboa.

Mateo, J., Ayres, C. \& López-Jurado, L. F. (2011). Los anfibios y reptiles naturalizados en España: historia y evolución de una problemática creciente. Boletín de la Asociación Herpetológica Española 22: 447-454.

Nevo, E., Gorman, G., Soulé, M., Yang, S. Y., Clover, R. \& Jovanovic, V. (1972). Competitive exclusion between insular Lacerta species (Sauria, Lacertidae). Oecologia (Berl.) 10: 183-190.

Ouboter, P. E. (1981). The ecology of the islandlizard Podarcis sicula salfii. AmphibiaReptilia 2(3): 243-257.

Pedrosa, J.P.F.L. (2013). Parque das Nações: abordagem precursora ao desenho da cidade sustentável. M.Sc. Thesis in Architecture, Faculdade de Ciências e Tecnologia da Universidade de Coimbra.

Pellitteri-Rosa, D., Liuzzi, C. \& Bellati, A. (2015). First report of adult Podarcis siculus (Rafinesque-Schmaltz, 1810) predation by Tarentola mauritanica (Linnaeus, 1758). Herpetozoa 28 (1/2): 89-92.

Podnar, M., Mayer, W. \& Tvrtković, N. (2005). Phylogeography of the Italian wall lizard, Podarcis sicula, as revealed by mitochondrial DNA sequences. Molecular Ecology 14(2): 575-588.

Ribeiro, R. (2017). Avaliação do estádio de introdução da lagartixa italiana Podarcis siculus no Parque das Nações (Lisboa). M.Sc. Thesis in Conservation Biology, Universidade de Évora.

Rivera, X., Arribas, O., Carranza, S. \& MaLuquer-Margalef, J. (2011). An introduction of Podarcis sicula in Catalonia (NE Iberian
Peninsula) on imported olive trees. Butlletí de la Societat Catalana d'Herpetologia 19: 79-85.

Rugiero, L. \& Luiselli, L. (2007). Null model analysis of lizard communities in five urban parks of Rome. Amphibia-Reptilia 28(4): 547553.

Schulte, U. (2012). Origin, climate niche, population genetics and intraspecific hybridization of introduced wall lizard populations in Central Europe (Ph.D dissertation, Universität Trier).

Senczuk, G., Colangelo, P., De Simone, E., Aloise, G., \& Castiglia, R. (2017). A combination of long term fragmentation and glacial persistence drove the evolutionary history of the Italian wall lizard Podarcis siculus. BMC evolutionary biology 17(6): 1-15.

Silva-Rocha, I., Salvi, D. \& Carretero, M. A. (2012): Genetic data reveal a multiple origin for the populations of the Italian wall lizard Podarcis sicula (Squamata: Lacertidae) introduced in the Iberian Peninsula and Balearic Islands. Italian Journal of Zoology 79(4): 502 -510 .

Silva-Rocha, I., Salvi, D., Harris, D. J., Freitas, S., Davis, C., Foster, J., Deichsel, G., Adamopoulou, C. \& Carretero, M. A. (2014). Molecular assessment of Podarcis sicula populations in Britain, Greece and Turkey reinforces a multiple-origin invasion pattern in this species. Acta Herpetologica 9(2): 253258.

Smith, G. R., Ballinger, R. E. (2001). The ecological consequences of habitat and microhabitat use in lizards: a review. Contemporary Herpetology 2001(3): 1-13.

Stamps, J. (1995). Motor learning and the value of familiar space. The American Naturalist 146(1): 41-58.

Strode, P. \& Brokaw, A. (2015). Using biointeractive resources to teach Mathematics and Statistics in Biology, In http:// media.hhmi.org/biointeractive/guides/

statistics/Statistics-Teacher Guide.pdf? download=true - Accessed at February 25th, 2017 
Valdeón, A., Perera, A., Costa, S., Sampaio, F. Evolution 24(3): 244-256.

\& Carretero, M. A. (2010). Evidencia de Vitt, L. J. \& Caldwell, J. P. (2014). Herpetology: una introducción de Podarcis sicula desde Italia a España asociada a una importación de olivos (Olea europaea). Boletín de la Asociación Herpetológica Española 21: 122-126.

Vignoli, L., Vuerich, V., \& Bologna, M. A. (2012). Experimental study of dispersal behaviour in a wall lizard species (Podarcis sicula) (Sauria Lacertidae). Ethology Ecology $\mathcal{E}$ an introductory biology of amphibians and reptiles. $4^{\text {th }}$ ed. Academic Press.

Žagar, A., Bitenc, K., Vrezec, A., \& Carretero, M. A. (2015). Predators as mediators: Differential antipredator behavior in competitive lizard species in a multi-predator environment. Zoologischer Anzeiger - A Journal of Comparative Zoology 259: 31-40. 